\title{
On the Core of Dynamic Cooperative Games
}

\author{
Ehud Lehrer* \\ School of Mathematical Sciences \\ Tel Aviv University \\ Tel Aviv 69978, Israel \\ and INSEAD \\ Bd. de Constance \\ F-77305 Fontainebleau Cedex, France. \\ lehrer@post.tau.ac.il \\ Marco Scarsini \\ Dipartimento di Economia e Finanza \\ LUISS \\ Viale Romania 12 \\ I-00197 Roma, Italy. \\ marco.scarsini@luiss.it
}

June 14, 2021

\footnotetext{
${ }^{*}$ This author acknowledges the support of the Israel Science Foundation, Grant \#762/045.
} 


\begin{abstract}
We consider dynamic cooperative games, where the worth of coalitions varies over time according to the history of allocations. When defining the core of a dynamic game, we allow the possibility for coalitions to deviate at any time and thereby to give rise to a new environment. A coalition that considers a deviation needs to take the consequences into account because from the deviation point on, the game is no longer played with the original set of players. The deviating coalition becomes the new grand coalition which, in turn, induces a new dynamic game. The stage games of the new dynamical game depend on all previous allocation including those that have materialized from the deviating time on.

We define three types of core solutions: fair core, stable core and credible core. We characterize the first two in case where the instantaneous game depends on the last allocation (rather than on the whole history of allocations) and the third in the general case. The analysis and the results resembles to a great extent the theory of non-cooperative dynamic games.
\end{abstract}

AMS 2000 Subject Classification: Primary 91A12, Secondary 91A25.

Journal of Economic Literature classification numbers: C71.

Keywords: fair core, stable core, credible core, convexification of a game. 


\section{Introduction}

Noncooperative game theory has dedicated a lot of attention to dynamic games and refinements of Nash equilibrium have been studied to capture the features that the dynamic induces in the game. When the dynamic is obtained by simply repeating a stage game over time, the folk theorem shows that the set of equilibria in an infinitely repeated game is in general much larger than the set of equilibria in the stage game.

In cooperative game theory most of the literature studies only static situations: a game is played only once and its solution is a set of suitable allocations that satisfies some conditions.

In this paper we consider a bona fide dynamic version of a cooperative game, where the worth of coalitions varies over time according to the history of the game. In particular the worth of coalitions at time $t$ depends on the allocations at all the times before $t$.

When defining a solution concept we allow the possibility for coalitions to deviate at any time and thereby to give rise to a new environment. When a coalition deviates, from that point on, the game is no longer played with the original set of players. The deviating coalition becomes the new grand coalition which, in turn, induces a new dynamic game. The stage games of the new dynamical game depend on all previous allocations, including those that have materialized from the deviating time on.

The existing literature on dynamic cooperative games considers games that determine only the worth of any coalition in a stage game played with the original grand coalition. However, in order to accommodate the possibility of deviating coalitions that generate new dynamical games, we need a richer structure. In the model of dynamic cooperative games that we introduce, the grand coalition of any stage game might be strictly smaller than the original grand coalition, while the allocation history is adapted accordingly.

In this paper we focus on dynamic games where the stage games are deterministically determined by the historical allocations. In these games a sequence of allocations uniquely induces a sequence of stage games. We investigate the core in three different approaches.

A coalition is said to be under-treated if the present value of its stage-shares is smaller than the present stage-worth of it. A sequence of stage-allocations is in the fair core if no sub-coalition is under-treated. Under the fair core approach, which is similar to that taken by the relevant literature, an under-treated coalition may complain but it cannot change the evolution of the game by abandoning the previous environment and creating a whole new game.

In the stable core on the other hand, the share of a coalition is compared to the opportunities it would have if it decided to deviate. A coalition is said to be dissatisfied with a sequence of allocations if, by quitting the original game, the coalition can form another dynamic game, with a smaller number of players, and afford better future allocations. A sequence of allocations is in the stable core if no coalition can 
deviate and get on its own a greater share than the one proposed by the sequence.

The stable core does not consider what the threat of a potential deviating coalition consists of. It might be that the sequence of allocations a coalition shows in order to substantiate its dissatisfaction, is itself prone to deviations. Thus, a threat of a coalition to deviate and obtain a certain sequence of allocations may be non-sustainable and therefore non-credible. The credible core requires that any better sequence it might generate on its own be credible. That is, any sequence of future allocations must itself be immune to deviations of smaller sub-coalitions that are also immune to deviations of smaller coalitions.

In all our analysis at every stage $t$, either no player deviates and therefore a game involving all the players of the previous stage $t-1$ is played, or a coalition deviates and creates its own game, which is a subgame of the previous one. Players are never allowed to establish a larger coalition once they have deviated and formed a smaller one. So new games can be created by splitting, but not by aggregation. We make this assumption since, without it the possible dynamics would be so general as not to produce any interesting result. Moreover the assumption allows to describe a huge spectrum of situations of relevance.

When a coalition $S$ deviates from the grand coalition $N$, we do not take into account what happens to the coalition $N \backslash S$. This is due to the fact that we are concerned with stability and therefore with conditions that guarantee that no deviation will actually materialize, no matter what the status of the abandoned coalition is.

\subsection{Existing literature}

Dynamic cooperative games have been studied in a few versions. Most of the studies, as we do, concentrate on the core. Oviedo (2000) studies the core of a finitely repeated discounted cooperative game where the stage game does not vary over time and no dynamic consideration is involved.

Kranich, Perea, and Peters (2005) consider a finite horizon of predetermined games. They study three different core concepts. The classical core assumes that coalitions planning to split off do so right at the beginning. This concept does not depend on the temporal structure of the game: the classical core coincides with the core of an induced static game. The strong sequential core, on the other hand, allows for deviations of coalitions at any stage of the game, but once a coalition deviates at some point, it must keep doing so from that time on. In the above two concepts deviations are not required to be credible, i.e., they could be blocked by some sub-coalition in the future. The weak sequential core is robust against credible coalitional deviations. The latter means that deviations are immune to deviations of sub-coalitions. The sub-coalition deviations can be themselves non-credible. Habis and Herings (2010) provide a correction of the above definition of weak sequential core.

Predtetchinski (2007) deals with infinite-horizon stationary cooperative games, where at each moment the game is in one of a finite number of states, that determines 
which instantaneous game is played at that moment. The states evolves according to an exogenous Markov chain and it does not depend on past allocations. The author considers the classical core and a version of the strong sequential core, and provides conditions for nonemptyness of the strong sequential core. Hellman (2008) focuses on the bargaining set of dynamic cooperative games, where the sequence of stage TU-games is exogenously specified.

Related results can be found in Gale (1978), where a concept of sequential core is defined and is used to model lack of trust in a two-period economy. In this model coalitions are allowed to deviate in the second period. Becker and Chakrabarti (1995) consider infinite horizon capital allocation models and define recursive core allocations, the ones where no coalition can improve upon its consumption stream at any time given its accumulation of assets up to that period.

Koutsougeras (1998) introduces the notion of two-stage core, that takes into account the possibility of temporary cooperation. Within each coalition agents make future trades only if they are enforceable, i.e., a coalition may have a limited horizon. Moreover a coalition blocks at some point in time only if it can secure improvements for its members in any possible consequence of a deviation. Predtetchinski, Herings, and Peters (2002); Predtetchinski, Herings, and Perea (2006) use the concepts of strong and weak sequential core in the context of two-period economies. Predtetchinski. Herings, and Peters (2004) apply the concept of strong sequential core to a stationary exchange economy.

Petrosian (1977, 1993) deals with a cooperative game induced by a (non-cooperative) differential game and Petrosjan and Zaccour (2003) study the problem of allocation over time of total cost incurred by countries in a cooperative game of pollution reduction and compute the Shapley value of this game. These papers are not about dynamic cooperative games but about a cooperative game induced by non-cooperative game played over time.

There exists a whole literature on coalition formation, where stability of coalitions is considered under different aspects (see, e.g., Rav, 1989; Chwe, 1994; Xue, 1998; Ray and Vohra, 1999; Konishi and Ray, 2003; Diamantoudi and Xue, 2007; Ray, 2007, and references therein). Typically this literature considers strategically richer models than the one examined in this paper, so it is closer in spirit to noncooperative game theory. For instance the model considered by Konishi and Ray (2003), which describes coalition formation as a truly dynamical process, considers a state space, beliefs, a probabilistic structure, and equilibrium concepts.

Our notion of credible core can be related to the papers by Bernheim, Peleg, and Whinston (1987); Bernheim and Whinston (1987) on coalition-proof Nash equilibria.

In some of our results we resort to the concept of $\varepsilon$-core. This was introduced in Shapley and Shubik (1966) to analyze situations where the core is empty. It has been employed in different contexts by Wooders (1983); Shubik and Wooders (1983a, b) ; Wooders and Zame (1984); Kovalenkov and Wooders (2001a.b, 2003, 2005), among others. In some of these papers a parametrized collection of cooperative games is considered and an approximate core is computed, where the goodness of the approx- 
imation depends on the parameters of the game. In particular, given the parameters $\pi$ describing a collection of games and given a lower bound $n_{0}$ on the number of players in each game in the collection, Kovalenkov and Wooders (2001b) obtain a bound $\varepsilon\left(\pi, n_{0}\right)$ so that, for any $\varepsilon \geq \varepsilon\left(\pi, n_{0}\right)$, all games in the collection with at least $n_{0}$ players have nonempty $\varepsilon$-cores. Some of our results have a similar flavor, except that for us the lower bound on the $\varepsilon$ is zero and the quantity that guarantees the existence of the $\varepsilon$-core is the discount factor, rather than the number of players.

The paper is organized as follows. In Section 2 we give a motivating example based on the classical market games of Shapley and Shubik. The model is introduced in 3 and the two first types of core solutions are given in Section 4. Section 5 provides characterizations of the non-emptyness of the $\varepsilon$-core when the discount factor is sufficiently large. The credible core is discussed in Section [6 and the paper ends with a section devoted to a few final remarks.

\section{A motivating example: A market with external- ities}

To show a typical application of dynamic cooperative games consider $n$ firms that engage repeatedly in a market game. At any period each firm brings into the market its own endowment and technology, that might depend on the firm's previous allocation. The firms then share their endowments in order to produce the maximal possible quantity. An important feature of the model is the existence of a positive externality reflected in the knowhow of each firm. The production function of each firm increases as the number of firms in the economy increases.

Formally, let $N=\{1,2, \ldots, n\}$ be the set of firms that are capable of producing a certain commodity using $\ell$ production factors. In the static version of the model, when the input of production factors is $y=\left(y_{1}, \ldots, y_{\ell}\right) \in \mathbb{R}_{+}^{\ell}$, firm $i$ produces $e(n) u^{i}(y)$, where $u^{i}$ is a concave function and $e(n)$ is the externality factor which is increasing with the number $n$ of firms in the economy.

The relevance of the externality factor becomes clear in the dynamic model. An under-treated coalition of firms might want to split off and form its own consortium. By doing so, on one hand, as an independent consortium, it will be subject to a smaller externality factor, since the number of cooperating firms is reduced. On the other hand, it will have the full freedom to share the entire profit the way it wishes.

To make the model more realistic, we assume that the production functions change over time and that, in order to keep the production ability, firms need to invest every period in maintenance, which requires resources. These resources come from the allocation of the firms in previous times, and whatever does not go into maintenance, is used for dividends. Thus, the current production function depends on yesterday's allocation and the externality factor. 
For instance, suppose that the production function of firm $i$ at time $t$ is

$$
u_{t}^{i}(y)=e(k) \gamma^{1 /\left(1+x_{t-1}^{i}\right)} u_{t-1}^{i}(y),
$$

where $k$ is the number of firms in the consortium that $i$ belongs to, $x_{t-1}^{i}$ is the allocation of firm $i$ at time $t-1$, and $0<\gamma<1$ is the decay rate per period. Note that $\gamma^{1 /\left(1+x_{t-1}^{i}\right)}$ is increasing with $x_{t-1}^{i}$ and therefore the greater the allocation at time $t-1$, the more efficient the firm at time $t$.

For the sake of simplicity assume that each firm is endowed anew at any time with the same production factor basket, say $y^{i}$. We are ready now to describe the dynamic. If at time $t$ firm $i$ belongs to the consortium $S$, then it engages the stage market game $v_{t}^{S}$ defined by

$$
v_{t}^{S}(T)=\max \left\{\sum_{i \in T} u_{t}^{i}\left(z^{i}\right) ; \quad \sum_{i \in T} z^{i}=\sum_{i \in T} y^{i}, z^{i} \in \mathbb{R}_{+}^{\ell}\right\}
$$

for every $T \subseteq S$, with $k=|S|$ in (2.1).

\section{Dynamic cooperative games}

\subsection{Dynamic of the game}

Let $N=\{1, \ldots, n\}$ be the set of players. For any coalition $S \subseteq N$ consider a function $v^{S}: 2^{S} \rightarrow \mathbb{R}_{+}$with $v^{S}(\varnothing)=0$. The function $v^{S}$ is called characteristic function defined over $S$ with the set $S$ being the grand coalition of $v^{S}$. An allocation of $v^{S}$ is a vector $x^{S} \in \mathbb{R}^{S}$ that satisfies $\sum_{i \in S} x^{S}(i)=v^{S}(S)$ and $x^{S}(i) \geq B$, where $B$ is a uniform lower bound over all allocations. The reason for this lower bound is primarily technical: with this lower bound the set of allocations becomes compact. If we take $B=0$, no inter-temporal loans are allowed, whereas, when $B<0$ a player can get less than her individually rational level at a certain stage, but then she will be compensated in the future.

At any stage $t$ a cooperative games over a grand coalition $S$ is played. Both the game and the grand coalition depend on the history up to that stage. The players of the grand coalition $S$ are getting at time $t$ an allocation of the game actually being played. The cooperative game of the subsequent period depends on the current allocation.

Formally, a dynamic cooperative game is played over a discrete set of periods. The evolution of the system depends on the initial game, the allocation at every period and the dynamic $V$, specified below. At stage 1 any coalition decides whether to split off or not. If no coalition splits off, then the initial cooperative game $v_{1}^{N}$ is played, and the allocation is $x_{1}^{N}$. If $S$ splits off, then the initial cooperative game is $v_{1}^{S}$ and the allocation is $x_{1}^{S}$. Note that the initial game is predetermined and is beyond the control of the players, unless a sub-coalition wishes to split off. 
Like in a static cooperative game we will use (different versions of) the core to determine whether the grand coalition $N$ is stable (in different senses), namely no sub-coalition $S$ has an incentive to split. In case a coalition $S$ can advantageously split, the grand coalition $N$ is not stable, which makes the core empty. That is why we do not need to specify what payoffs players in $N \backslash S$ get or whether some of them want to form a coalition of their own.

From period 2 on, the evolution is governed by $V$. The state of the system is a pair $\left(S ; x^{S}\right)$, where $S$ is a coalition and $x^{S}$ is an allocation of $S$. As long as no coalition splits off, the state is of the form $\left(N ; x^{N}\right)$; once a coalition $S \subseteq N$ deviates, the system turns to a state of the type $\left(S ; x^{S}\right)$, and $S$ remains fixed from that stage on forever.

When the state at time $t$ is $\left(N ; x^{N}\right)$, unless a deviation of $S$ occurs, the game played at time $t+1$ is $V\left(N ; x^{N}\right)$, whose grand coalition is $N$. At this time the allocation is $x_{t+1}^{N}$. However, if $S$ deviates, the game played at time $t+1$ is $V\left(S ; x_{S}^{N}\right)$, where $x_{S}^{N}$ is the allocation induced by $x^{N}$ to coalition $S$. At time $t+1$ the allocation is $x_{t+1}^{S}$ and the subsequent game is $V\left(S ; x_{t+1}^{S}\right)$ whose grand coalition is $S$.

For the sake of simplicity we assume a Markovian structure of the game, where the stage game played at time $t$ depends on the allocation at time $t-1$. More complicated dynamics could be considered, for instance the game at time $t$ could depend on the whole past history. An interesting intermediate case is the one where the game depends on some unidimensional function of the history, for instance on the sum of the past allocations. Think for instance of a model of dynamic public good provision, where every player contributes to a public good, whose level at time $t$ depends on the (discounted) sum of past contributions.

A few remarks regarding our modeling choices are in place. Up to Section 6, we assume that once a coalition deviates, it remains the grand coalition forever and no further splitting off of sub-coalitions will take place. This restriction corresponds to the first two types of core solutions, that are concerned with long-term plans that prevent these kind of deviations. When dealing with the third type of core solution we lift this restriction. A deviating coalition is not protected against coups of its sub-coalitions. This is the reason why a threat of a coalition to deviate is rendered credible only if it is immunized against further split offs of its sub-coalitions.

We also assume here that once a coalition deviates, there will be no way to restore a full cooperation and to rebuild the grand coalition $N$. Such a possibility requires a much more complicated dynamic that would depend also on past allocations of non-deviating members. In this paper we decide to keep matters as simple as possible. This distinguishes our model from the literature on coalition formation that we mentioned in the Introduction.

In our model a coalition $S$ can deviate prior to time 1 and play the game $v_{1}^{S}$. We could as well assume that at time 1 for any $S, V\left(N, x_{0}^{N}\right)(S)=V\left(S, x_{0}^{N}\right)(S)$, and it will make no difference in the results. Note however, that in the motivating example, when the grand coalition is $N$, the worth of $S$ is typically different from its worth 
when the grand coalition is $S$.

Our model refers to the deviating coalition, but it ignores the rest of the players. In principle, the complement of a deviating coalition could be treated just like the deviating coalition itself. The continuation game of the complement depends on its historical allocations. However, we chose not to specify it for two reasons. First, our study focuses on two main aspects, fairness and stability. Whether or not a coalition is treated equitably does not depend on what happens to its complement. The same applies to the willingness of a coalition to split off: it is not affected by the its complement.

The second reason is that we study core solutions and characterize the games whose core is non-empty. In these games no deviation will occur and in any case no coalition will be left alone without its complement.

\subsection{Discounting future payoffs}

We assume throughout that all players have the same discount factor $0<\delta<1$. Suppose that player $i$ 's payoff at time $t$ is $x_{t}(i)$. Her present normalized payoff is

$$
x_{*}(i, \delta)=(1-\delta) \sum_{t=1}^{\infty} \delta^{t-1} x_{t}(i) .
$$

We define for every $T \subseteq N$

$$
x_{*}(T, \delta)=\sum_{i \in T} x_{*}(i, \delta)
$$

Therefore $x_{*}(T, \delta)$ is the sum of all individual allocations of $T$ 's members.

Define a new characteristic function on $N$ as follows.

$$
v_{*}(S, \delta)=\max x_{*}(S, \delta)
$$

where the maximum is taken over all feasible histories of $S$-allocations: $x_{1}^{S}, x_{2}^{S}, \ldots$ and $x_{t}(i)=x_{t}^{S}(i)$.

The dynamic game with discount factor $\delta$ will be denoted by $(V, \delta)$.

\section{The fair and stable core solutions}

\subsection{The fair core}

There are two justifications for the definition of core in the classical model of one-shot cooperative game. The first is fairness and the second is stability. An allocation is in the core if any coalition obtains at least its worth. Behind this justification lies an assumptions that a central planner has a full control on what the players get, and 
once she makes up her mind regarding the split of the cake, the players have no way to protest.

This kind of reasoning leads us to define what we call the 'fair core' first. A sequence of allocations $x_{1}^{N}, x_{2}^{N}, \ldots$ is in the fair core if fore every coalition $S$ the present value of the shares of $S$ exceeds the present value of the worths of $S$. It is assumed that coalition $S$ can do nothing about its future shares, which gives the central planner the freedom to choose allocations without paying attention to semistrategic considerations like stability.

The definition of fair core is concerned solely with the following consideration: it is fair to give coalition $S$ allocations whose present value is no less than what their present worth is.

Definition 4.1 (Fair core). (i) A sequence of $N$-allocations $x_{1}^{N}, x_{2}^{N}, \ldots$ is in the fair core of $(V, \delta)$ if for every $S \subseteq N$

$$
x_{*}^{N}(S, \delta) \geq(1-\delta) \sum_{t=1}^{\infty} \delta^{t-1} V\left(N ; x_{t-1}^{N}\right)(S) .
$$

(ii) A sequence of $N$-allocations $x_{1}^{N}, x_{2}^{N}, \ldots$ is in the $\varepsilon$-fair core of $(V, \delta)$ if for every $S \subseteq N$

$$
x_{*}^{N}(S, \delta) \geq(1-\delta) \sum_{t=1}^{\infty} \delta^{t-1} V\left(N ; x_{t-1}^{N}\right)(S)-\varepsilon .
$$

If the core of the stage game at time $t$ is nonempty, for every $t$, then the fair core of $(V, \delta)$ is nonempty.

Example 4.2. It is possible for a game $(V, \delta)$ to have a nonempty fair core even if for every $t$ the core at the stage game is empty. Consider the four player games $u_{1}$ and $u_{2}$, where $u_{1}(i)=0$ for every player $i, u_{1}(12)=u_{1}(23)=u_{1}(13)=3, u_{1}(123)=4$ and player 4 is dummy; game $u_{2}$ is like the $u_{1}$ where players 1 and 4 exchange their roles. The cores of $u_{1}$ and $u_{2}$ are empty.

Suppose that $v_{1}^{N}=u_{1}, V\left(N ;\left(1, \frac{3}{2}, \frac{3}{2}, 0\right)\right)=u_{2}$ and $V\left(N ;\left(0, \frac{3}{2}, \frac{3}{2}, 1\right)\right)=u_{1}$. Suppose that $x_{t}^{N}=\left(1, \frac{3}{2}, \frac{3}{2}, 0\right)$ when $t$ is odd and $x_{t}^{N}=\left(0, \frac{3}{2}, \frac{3}{2}, 1\right)$ when $t$ is even. The result is that $u_{1}$ is played in odd times and $u_{2}$ in even times.

When the discount factor $\delta$ is high, the average over time of stage games is close to $\left(u_{1}+u_{2}\right) / 2$ and the discounted value of the stream of allocations is close to $\left(\frac{1}{2}, \frac{3}{2}, \frac{3}{2}, \frac{1}{2}\right)$. Thus the sequence of payoffs $\left(x_{t}^{N}\right)_{t}$ is in the $\varepsilon$-fair core of $(V, \delta)$.

Definition 4.3 (Efficiency). A sequence of $N$-allocations $x_{1}^{N}, x_{2}^{N}, \ldots$ is efficient in $(V, \delta)$ if

$$
x_{*}^{N}(N, \delta) \geq v_{*}(N, \delta) .
$$


That is, $x_{1}^{N}, x_{2}^{N}, \ldots$ is efficient if the present value of the grand coalition's share is the maximum available. Recall that the actual game is history dependent. While any of the stage allocations could be locally efficient, it might reduce the size of the cake in subsequent periods and thereby might hamper efficiency.

In the classical one-shot game the definition of allocation contains the requirement of efficiency. This is not the case in the dynamic game. The fair core is not necessarily efficient, as demonstrated by the following example.

Example 4.4. Suppose that

$$
v_{1}(N ; S)= \begin{cases}1 & S=N \\ 0 & \text { otherwise }\end{cases}
$$

and, if the allocation $x^{N}$ is uniform (i.e., treats all players equally), then $V\left(N ; x^{N}\right)=$ $\left|x^{N}\right| v_{1}^{N}$, where $\left|x^{N}\right|$ stands for the sum of the individual allocations of all players, otherwise $V\left(N ; x^{N}\right)=0$. Call $e_{1}=(1,0, \ldots, 0) \in \mathbb{R}^{N}$ the first vector of the standard basis and $\overrightarrow{0}=(0, \ldots, 0) \in \mathbb{R}^{N}$ the zero vector. The sequence of allocations $e_{1}, \overrightarrow{0}, \overrightarrow{0}, \ldots$ is in the fair core: the first allocation $e_{1}$ is in the core of the stage game $v_{1}^{N}$, but it is not uniform and therefore all subsequent games are identically 0 . This sequence is in the fair core of $(V, \delta)$, but it is certainly not efficient.

Definition 4.5 (Efficient fair core). (i) A sequence of $N$-allocations $x_{1}^{N}, x_{2}^{N}, \ldots$ is in the efficient fair core of $(V, \delta)$ if it is efficient and in the fair core of $(V, \delta)$.

(ii) A sequence of $N$-allocations $x_{1}^{N}, x_{2}^{N}, \ldots$ is in the $\varepsilon$-efficient fair core of $(V, \delta)$ if it is efficient and in the $\varepsilon$-fair core of $(V, \delta)$.

In the special case where the worth of the grand coalition is constant, that is when the worth of the grand coalition in all stage games do not depend on the history of allocation nor on the time, the fair core and the efficient fair core coincide.

\subsection{The stable core}

The definition of the fair core does not make use of the entire structure of the dynamic game. It uses only states of the type $\left(N ; x^{N}\right)$ and not of the type $\left(S ; x^{S}\right)$, where $S \varsubsetneqq N$.

The second justification of the core involves stability considerations. An undertreated coalition might deviate, create its own game, and improve its position by reallocating its endowment. When a coalition $S$ threatens to deviate, it shifts the system to a state of the form $\left(S ; x^{S}\right)$ and the dynamic then is governed by $V(S ; \cdot)$. This is why referring just to states of the type $\left(N ; x^{N}\right)$ is insufficient and there is a need to refer to states of the form $\left(S ; x^{S}\right)$ for every coalition $S$. 
Let $x_{1}^{N}, x_{2}^{N}, \ldots$ be a sequence of allocations. Define

$$
x_{*}^{h}(i, \delta)=(1-\delta) \sum_{t=h}^{\infty} \delta^{t-1} x_{t}(i) .
$$

The number $x_{*}^{h}(i, \delta)$ represents the discounted value at time $h$ of the shares that player $i$ receives at time $h$ and on. In particular, $x_{*}(i, \delta)=x_{*}^{1}(i, \delta)$. Similarly, define the game $v_{*}^{h}(\cdot, \delta)$ as

$$
v_{*}^{h}(S, \delta)=\max \sum_{i \in S} x_{*}^{h}(i, \delta),
$$

where the maximum is taken over all feasible sequences $x_{1}^{N}, x_{2}^{N}, \ldots x_{h}^{N}, z_{h+1}^{S}, z_{h+2}^{S} \ldots$ that coincide with $x_{1}^{N}, x_{2}^{N}, \ldots$ up to $h$, the time when $S$ decides to leave.

A sequence of allocations $x_{1}^{N}, x_{2}^{N}, \ldots$ is in the stable core if at any time $h$ the value of the shares of coalition $S$ exceeds the value of what coalition $S$ could guarantee in autarky, meaning without being engaged with others.

Definition 4.6 (Stable core). (i) A sequence of $N$-allocations $x_{1}, x_{2}, \ldots$ is in the stable core of $(V, \delta)$ if for every $S \subseteq N$ and every $h$,

$$
x_{*}^{h}(S, \delta) \geq v_{*}^{h}(S, \delta) .
$$

(ii) A sequence of $N$-allocations $x_{1}^{N}, x_{2}^{N}, \ldots$ is in the $\varepsilon$-stable core of $(V, \delta)$ if for every $S \subseteq N$ and every $h$,

$$
x_{*}^{h}(S, \delta) \geq v_{*}^{h}(S, \delta)-\varepsilon .
$$

Remark 4.7. (a) Unlike the fair core, any history of $N$-allocations in the stable core is efficient.

(b) The two notions of core are not necessarily co-variant with linear transformations. If $V$ is co-variant with linear transformations, so are the two cores.

Example 4.8. It is possible for a game $(V, \delta)$ to have a nonempty stable core even if for every $t$ the core of the stage game is empty. Consider a set $N=\{1,2, \ldots, 2 k+1\}$, and let

$$
V\left(N ; x^{N}\right)(S)= \begin{cases}x^{N}(N) & \text { if }|S| \geq k+1, \\ 0 & \text { otherwise. }\end{cases}
$$

be $x^{N}(N)$ times a majority game, and let $V\left(S ; x^{S}\right)(T)=2^{-|T|} V(N ; \cdot)(T)$ for every $S \varsubsetneqq N$.

The core of each stage game $V\left(N ; x_{t}^{N}\right)$ is empty, and so is the fair core of $(V, \delta)$, whereas its stable core is not, regardless of the discount factor. The reason being that when a coalition deviates, its future payoff declines rapidly. Coalitions will be satisfied with shares that are strictly smaller than their worth, because deviation does not promise a greater portion. 


\section{Non-emptyness of the core}

\subsection{Non-emptyness of the fair core}

The following result applies to games where $v\left(N ; x^{N}\right)(N)$ is equal to $x^{N}(N)$ for every allocation $x^{N}$.

For the next definition we recall that the set of characteristic functions is a vector space. Denote by $\Delta(d)$ the set of stage allocations of games where the worth of the grand coalition is $d$. That is, $\Delta(d)=\left\{x^{N} ; x^{N}(N)=d, x^{N}(i) \geq B\right.$ for every $\left.i \in N\right\}$. The set $\Delta(d)$ is obviously compact, which is the reason why we impose the constraint that $x^{N}(i) \geq B$ for every $i \in N$.

Let $x \in \Delta(d)$ be an allocation. We say that $\left(y_{1}, \ldots, y_{k} ; \alpha_{1}, \ldots, \alpha_{k}\right)$ is a split of $x$, if

$$
x=\sum_{j=1}^{k} \alpha_{j} y_{j},
$$

where $\alpha_{j} \geq 0, \sum_{j=1}^{k} \alpha_{j}=1$, and $y_{j}$ is an allocation $j=1, \ldots, k$. That is, $x$ is a convex combination of the allocations $y_{j} \in \Delta(d)$ with $\alpha_{j}$ being the respective weights.

Definition 5.1. The convexification of $V(N ; \cdot)$, denoted conv $V(N ; \cdot)$, is a correspondence defined as follows. Let $x \in \Delta(d)$ be an allocation. Then, $\operatorname{conv} V(N ; x)$ is the set of all games that can be expressed as $\sum_{j=1}^{k} \alpha_{j} V\left(N, y_{j}\right)$, where $\left(y_{1}, \ldots, y_{k} ; \alpha_{1}, \ldots, \alpha_{k}\right)$ is a split of $x$.

If $V(N ; \cdot)$ is continuous, then conv $V(N ; x)$ is closed and therefore, conv $V(N ; x)$ also contains all games of the form $\sum_{j=1}^{\infty} \alpha_{j} V\left(N ; y_{j}\right)$, where $x$ is expressed as an infinite convex combination of allocations: $x=\sum_{j=1}^{\infty} \alpha_{j} y_{j}$.

Theorem 5.2. Consider a game where $V(N, \cdot)(N)=d$ and $V(N ; \cdot)$ is continuous. Assume that for every coalition $S, V(N ; \cdot)(S)$ is bounded. For every $\varepsilon>0$ there is $0<\delta_{0}<1$ such that for every $\delta \in\left(\delta_{0}, 1\right)$ the $\varepsilon$-fair core of $(V, \delta)$ is not empty if and only if there exists $x \in \Delta(d)$ and $v \in \operatorname{conv} V(N ; x)$ such that $x$ is in the core of $v$.

Before we get to the proof we wish to comment on the contents of this theorem. Just like in the folk theorem of the non-cooperative game theory, it characterizes the solution of the dynamic game in static terms. Specifically, it characterizes when the $\varepsilon$-fair core of the dynamic game is not empty in terms of the convexification of $V(N ; \cdot)$.

Note that we refer to the $\varepsilon$-fair core rather than to the fair core. The question is whether we do it because we just cannot prove anything stronger, or that it is due to a structural insurmountable difficulty. Recall that the dynamic game is described by the dynamics, $V$ and by an initial game played at the first stage. While there is some control of future games through past allocations, there is no way to alter the initial game. It might happen that this stage-game hinders the existence of an exact core while $\varepsilon$-fair core does exist. 
Proof of Theorem 5.2. Suppose first that for every $\varepsilon>0$ there is $1>\delta_{0}>0$ such that for every $\delta \in\left(\delta_{0}, 1\right)$ there is a sequence $x_{1}, x_{2}, \ldots$ of allocations in the $\varepsilon$-fair core of $(V, \delta)$.

Denoting,

$$
x=(1-\delta) \sum_{t=1}^{\infty} \delta^{t-1} x_{t} \quad \text { and } \quad u=(1-\delta) \sum_{t=1}^{\infty} \delta^{t-1} V\left(N ; x_{t-1}\right),
$$

we have for every $S \subseteq N$,

$$
x(S) \geq(1-\delta) \sum_{t=1}^{\infty} \delta^{t-1} V\left(N ; x_{t-1}\right)(S)-\varepsilon=u(S)-\varepsilon .
$$

Here, $V\left(N ; x_{0}\right)$ denotes the initial game, $v_{1}$.

Note that $(1-\delta) \sum_{t=2}^{\infty} \delta^{t-1} V\left(N ; x_{t-1}\right)=u(S)-(1-\delta) v_{1}$, and we obtain

$$
\begin{aligned}
x(S) & \geq u(S)-(1-\delta) v_{1}(S)+(1-\delta) v_{1}(S)-\varepsilon \\
& =(1-\delta) \sum_{t=2}^{\infty} \delta^{t-1} V\left(N ; x_{t-1}\right)+(1-\delta) v_{1}(S)-\varepsilon \\
& \geq(1-\delta) \sum_{t=2}^{\infty} \delta^{t-1} V\left(N ; x_{t-1}\right)-\varepsilon .
\end{aligned}
$$

When $\delta$ is sufficiently close to 1 , since $V(N ; \cdot)(S)$ is bounded, we have

$$
\delta \frac{1-\delta}{\delta} \sum_{t=2}^{\infty} \delta^{t-1} V\left(N ; x_{t-1}\right)(S)>\frac{1-\delta}{\delta} \sum_{t=2}^{\infty} \delta^{t-1} V\left(N ; x_{t-1}\right)(S)-\varepsilon
$$

Thus,

$$
\begin{aligned}
x(S) & \geq(1-\delta) \sum_{t=2}^{\infty} \delta^{t-1} V\left(N ; x_{t-1}\right)-\varepsilon \\
& =\delta \frac{1-\delta}{\delta} \sum_{t=2}^{\infty} \delta^{t-1} V\left(N ; x_{t-1}\right)(S)-\varepsilon \\
& \geq \frac{1-\delta}{\delta} \sum_{t=2}^{\infty} \delta^{t-1} V\left(N ; x_{t-1}\right)(S)-2 \varepsilon .
\end{aligned}
$$

Define

$$
x^{\prime}=\frac{1-\delta}{\delta} \sum_{t=2}^{\infty} \delta^{t-1} x_{t}
$$


If $\delta$ is sufficiently large, for every $S,\left|x(S)-x^{\prime}(S)\right|<\varepsilon$, and therefore,

$$
x^{\prime}(S) \geq \frac{1-\delta}{\delta} \sum_{t=2}^{\infty} \delta^{t-1} V\left(N ; x_{t-1}\right)(S)-3 \varepsilon .
$$

In other words, $x^{\prime}$ is in the $3 \varepsilon$-core of the game

$$
\frac{1-\delta}{\delta} \sum_{t=2}^{\infty} \delta^{t-1} V\left(N ; x_{t-1}\right)
$$

which is a (infinite) convex combination of the games $V\left(N ; x_{t-1}\right), t=2, \ldots$, each with the weight

$$
\delta^{t-1} \frac{1-\delta}{\delta}=\delta^{t-2}(1-\delta)
$$

and is therefore in conv $V\left(N ; x^{\prime}\right)$ (because $x^{\prime}$ is a convex combination of $x_{t-1}$ 's with the weights $\left.\delta^{t-2}(1-\delta), t=2,3, \ldots\right)$.

Since $\varepsilon$ is arbitrary, from compactness and continuity of $V(N ; \cdot)$, we conclude that there exists $v \in \operatorname{conv} V(N ; x)$ such that $x$ is in the core $v$, as desired.

We now assume that there is a vector $x \in \Delta$ such that $x$ is in the core of $v \in$ conv $V(N ; x)$. By definition of conv $V(N ; x)$, there is a split $\left(y_{1}, \ldots, y_{k} ; \alpha_{1}, \ldots, \alpha_{k}\right)$ of $x$ such that

$$
\alpha_{1} V\left(N ; y_{1}\right)+\cdots+\alpha_{k} V\left(N ; y_{k}\right)=v .
$$

Fix an $\varepsilon>0$. For $\delta$ large enough one can divide the set of periods into $k$ disjoint sets $T^{1}, \ldots, T^{k}$ in a way that for every $j=1, \ldots, k$,

$$
\alpha^{j}=(1-\delta) \sum_{t \in T^{j}} \delta^{t-1}
$$

(see for instance, Fudenberg and Maskin (1986)).

At time 1 set $v_{1}$ as an arbitrary game where $v_{1}(N)=d$. And in general, for $t \in T^{j}$ define $x_{t}=y_{j}$. In words, over the time periods in the set $T^{j}$ the allocation is $y_{j}$ and the game that follows is $V\left(N ; y_{j}\right)$. Note that since $V\left(N ; y_{j}\right)(N)$ is fixed and equal to $d$, for every $i=1, \ldots, k, y_{i}$ is an allocation of $V\left(N ; y_{j}\right)$ (because $y_{i} \in \Delta(d)$ ). By construction and (5.1) the present value of allocations is $x$.

On the other hand, the present value of all the stage games is

$$
(1-\delta) v_{1}+\sum_{j=1}^{k}(1-\delta) \sum_{t \in T^{j}, t \neq 1} \delta^{t-1} V\left(N ; x_{t-1}\right)=(1-\delta) v_{1}+\sum_{j=1}^{k}(1-\delta)\left[\sum_{t \in T^{j}, t \neq 1} \delta^{t-1}\right] V\left(N ; y_{j}\right) .
$$


Let $j_{0}$ be such that $1 \in T^{j_{0}}$. Using (5.3) we obtain for every coalition $S$,

$$
\begin{aligned}
& (1-\delta) v_{1}(S)+\sum_{j=1}^{k}(1-\delta) \sum_{t \in T^{j}, t \neq 1} \delta^{t-1} V\left(N ; x_{t-1}\right)(S) \\
& =(1-\delta) v_{1}(S)+\sum_{j=1}^{k} \alpha_{j} V\left(N ; y_{j}\right)(S)-(1-\delta) V\left(N ; y_{j_{0}}\right)(S) \\
& \leq(1-\delta) v_{1}(S)+\sum_{j=1}^{k} \alpha_{j} V\left(N ; y_{j}\right)(S)
\end{aligned}
$$

As $V(N ; \cdot)$ is continuous, $V(N ; x)(S)$ is bounded and therefore, when $\delta$ is sufficiently close to $1,(1-\delta) v_{1}(S)<\varepsilon$ for every coalition $S$. Thus, the present value for every coalition $S$ satisfies,

$$
\left((1-\delta) v_{1}+\sum_{j=1}^{k}(1-\delta) \sum_{t \in T^{j}, t \neq 1} \delta^{t-1} V\left(N ; x_{t-1}\right)\right)(S) \leq v(S)+\varepsilon .
$$

Since $x$ is in the core of $v$, the sequence $x_{1}, x_{2}, \ldots$ is in the $\varepsilon$-fair core of the dynamic game $V^{N}$ with the discount factor $\delta$.

Example 5.3. Let $N=\{1,2,3\}$ and let $e_{i}$ be the $i$-th vector of the standard basis in $\mathbb{R}^{3}$, that is, its $i$-th coordinate is 1 and the others are 0 . Define $V\left(N, e_{i}\right)$ to be the additive game where $v(j)=1 / 2$ for $j \neq i$ and $v(i)=0$, which we denote as $p_{-i}$. When $x=\left(x^{1}, x^{2}, x^{3}\right)$ is such that $x^{i} \leq 4 / 5$ for every $i \in N$, then $V(N, x)$ is the simple majority game (i.e., $V\left(N, x_{0}^{N}\right)(S)=1$ iff $|S| \geq 2$ ). Moreover $V(N, \cdot)$ is extended to the whole simplex in a continuous fashion, keeping $V(N, \cdot)(N)=1$. Note that in all the games involved, the feasible allocations are elements of the simplex $\left\{\left(x^{1}, x^{2}, x^{3}\right): x^{i} \geq 0, \sum_{i} x^{i}=1\right\}$.

Let $v_{1}$, the initial game, be the simple majority game. Set, $x_{1}=e_{1}, x_{2}=e_{2}$ and $x_{t}=e_{t(\bmod 3)}$. The dynamic induces: $v_{2}=V\left(N, x_{1}\right)=p_{-1}, v_{3}=V\left(N, x_{2}\right)=p_{-2}$,

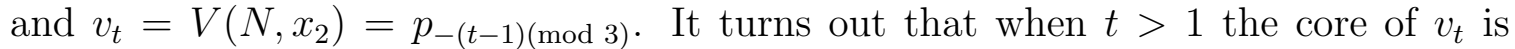
non-empty. However, if the allocation at time $t$ is the unique core allocation of $v_{t}$, the next stage-game is the majority game, whose core is empty.

It is easy to check that $x_{1}, x_{2}, \ldots$ is in the $\varepsilon$-fair core for discount factors large enough. To see that the dynamical game satisfies the sufficient condition of Theorem 5.2, consider $x=(1 / 3,1 / 3,1 / 3)$. Let $v$ be the additive game with weights $1 / 3$ assigned to each player. Note that

(a) $x$ is in the core of $v$;

(b) $\sum_{i=1}^{3} \frac{1}{3} e_{i}$ is a split of $x$ and

\footnotetext{
${ }^{1}$ Here, $3 k(\bmod 3)=3$.
} 
(c) $v=\sum_{i=1}^{3} \frac{1}{3} p_{-i}=\sum_{i=1}^{3} \frac{1}{3} V\left(N, e_{i}\right)$.

Thus the sufficient condition of Theorem 5.2 is satisfied.

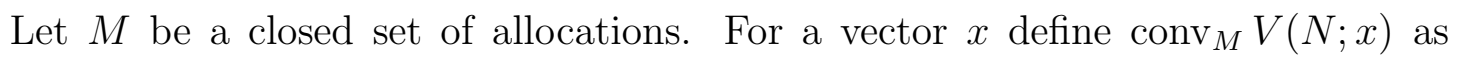
conv $V^{N}(x)$ was defined, with the extra condition that the allocations $y_{j}$ are in conv $M$. That is, $\operatorname{conv}_{M} V(N ; x)$ is the set all games that can be expressed as $\sum_{j=1}^{k} \alpha_{j} V\left(N ; y_{j}\right)$, where $\left(y_{1}, \ldots, y_{k} ; \alpha_{1}, \ldots, \alpha_{k}\right)$ is a split of $x$ and $y_{j} \in \operatorname{conv} M$, $j=1, \ldots, k$.

Theorem 5.4. Consider a game where $V(N ; \cdot)$ is continuous and bounded. For $\gamma>0$ denote $M_{\gamma}=\left\{x ; V(N ; x)(N)>\sup _{y} V(N ; y)(N)-\gamma\right\}$. For any $\varepsilon>0$ the efficient $\varepsilon$-fair core of a game $V(N ; \cdot)$ is not empty for $\delta$ large enough if and only if for every $\gamma$ sufficiently small there exists $x$ and $v \in \operatorname{conv}_{M_{\gamma}} V(N ; x)$ such that $x$ is in the $\gamma$-core of $v$.

The proof is similar to the proof of Theorem 5.2 and is therefore omitted.

\subsection{Non-emptyness of the stable core}

Recall that $V\left(S ; x^{S}\right)$ is the stage game played with $S$ as the grand coalition after a stage in which the allocation was $x^{S}$. We now assume that $V(S ; x)(T)$ depends on $x^{S}(T)=\sum_{i \in T} x_{i}$ for every $S$ that contains $T$ in a continuous and monotonically increasing fashion. In particular, the worth of coalition $T \subseteq S$ at time $t$ depends only on its total share at time $t-1$. For every coalition $T$ and time $t$, we define $U_{T}^{t}(c)$, inductively. $U_{T}^{1}(c)=V(S ; x)(T)$, where $x(T)=c$. Note that this is well defined, as $V(S ; x)(T)$ depends solely on $x(T)$. Then, $U_{T}^{t}(c)=U_{T}^{1}\left(U_{T}^{t-1}(c)\right)$. Define $f_{T}(c)$ to be the limit of $U_{T}^{t}(c)$. Due to continuity this limit exists. Thus, it satisfies $f_{T}(c)=f_{T}\left(f_{T}(c)\right)$. That is, $f_{T}(c)$ is a fixed point of $U_{T}^{1}$ and of $f_{T}$.

We further assume that $f_{T}(c)$ is finite for every $T$ and $c$, which in equivalent to assuming that either the set of fixed points of $U_{T}^{1}$ is unbounded or $U_{T}^{1}(x)<x$ for $x$ sufficiently large 2

Let $x$ be an allocation of $v_{1}$ and define the characteristic function $u_{x}$ as follows: $u_{x}(T)=f_{T}(x(T))$ for every coalition $T$.

Lemma 5.5. For every $\varepsilon>0$ there exists a time $m$ such that for every $c \leq v_{1}(N)$, $T \subseteq N$ and an allocation $x$ of $v_{1}$ with $x(T)=c,\left|u_{x}(T)-U_{T}^{t}(c)\right|<\varepsilon$ for every $t \geq m$.

\footnotetext{
${ }^{2}$ The function $U_{T}^{1}$ is continuous and monotonic. In case the set of the fixed points of $U_{T}^{1}$ is unbounded, every non-fixed point of $U_{T}^{1}$ is between two fixed points. The set of fixed points of $U_{T}^{1}$ is closed, and therefore for every non-fixed point of $U_{T}^{1}$, say $c$, there are two closest fixed point, one above $c$ and one below it. The sequence $U_{T}^{t}(c)$ then converges to one of the two (depending on whether $U_{T}^{t}(c)>c$ or $U_{T}^{t}(c)<c$ ). If, however, the set of fixed points of $U_{T}^{1}$ is bounded, then the sequence $U_{T}^{t}(c)$ diverges to infinity in case $U_{T}^{t}(c)>c$ asymptotically. In case $U_{T}^{t}(c)<c$ asymptotically, the sequence $U_{T}^{t}(c)$ is decreasing and $f_{T}(c)$ is finite.
} 
Proof. Fix $\varepsilon>0$ and a coalition $T$. Denote by $F$ the set of fixed points of $V(S ; x)(T)$ in the interval $\left[0, v_{1}(N)\right]$. Let $B$ be a finite subset of $F$ having the property that for every $a \in F$ there is $b \in B$ such that $|a-b|<\varepsilon$. Since $V(S ; x)(T)$ is continuous and monotonically increasing in $x(T), f_{T}$ is monotonically increasing. Thus, $f_{T}(a) \in$ $\left[b_{1}, b_{2}\right]$, for every $a \in\left[b_{1}, b_{2}\right]$ with $b_{1}, b_{2} \in B$. Moreover, the distance $\left|f_{T}(a)-U_{T}^{t}(a)\right|$ is decreasing with $t$. Denote by $A(b)$ the set of all points that are absorbed to $b \in B$. That is, $A(b)=\left\{a: f_{T}(a)=b\right\}$.

For every $b \in B$, there is time $m_{b}$ such that $\left|b-U_{T}^{t}(a)\right|<\varepsilon$ for every $t \geq m_{b}$ and $a \in A(b)$. Let $m_{T}=\max \left\{m_{b}: b \in B\right\}$. Thus, for every $a \in\left[0, v_{1}(N)\right]$, either $a \in A(b)$ for some $b \in B$, in which case $\left|f_{T}(a)-U_{T}^{t}(a)\right|=\left|b-U_{T}^{t}(a)\right|<\varepsilon$ for every $t \geq m_{T}$, or $\left|a-f_{T}(a)\right| \leq\left|U_{T}^{t}(a)-f_{T}(a)\right|<\varepsilon$ for every $t$. Since there are finitely many coalitions, $m=\max \left\{m_{T}: T \subseteq N\right\}$ satisfies the assertion of the lemma.

Theorem 5.6. Consider a game where $V(S ; x)(T)$ is continuously determined by $x(T)$ in an increasingly monotonic fashion. Assume furthermore, that $V(N ; x)(N)=$ $v_{1}(N)=1$ when $x(N)=v_{1}(N)$. Then, the two following statements are equivalent:

(i) For any $\varepsilon>0$ there is $\delta_{0}<1$ such that for every $\delta \in\left[\delta_{0}, 1\right)$ the $\varepsilon$-stable core of a game $(V, \delta)$ is not empty.

(ii) For every $\varepsilon>0$ there exists an allocation $x$ of $v_{1}$ such that the $\varepsilon$-core of $u_{x}$ is not empty.

Before we proceed to the proof of this theorem, we need an auxiliary result. Let $a_{1}, a_{2}, \ldots$ be a bounded sequence of numbers. For any integer $h$ denote, $a_{*}^{h, \delta}=$ $(1-\delta) \sum_{t=h}^{\infty} \delta^{t-h} a_{t}$. The proof of Theorem 5.6 uses the following lemma.

Lemma 5.7. For every $\delta<1$ large enough and every bounded sequence of numbers $a_{1}, a_{2}, \ldots$ such that $\left\{a_{*}^{h, \delta}\right\}_{h}$ has an accumulation point $a \geq 0$, and every $\gamma>0$ there is a time $h$ such that $a_{h}>a-\gamma$, while $a_{*}^{h}<a+\gamma$.

Proof. For every $h_{1}<h_{2}$,

$$
a_{*}^{h_{1}, \delta}=(1-\delta) \sum_{t=h_{1}}^{h_{2}-1} \delta^{t-h_{1}} a_{t}+(1-\delta)\left(1-\sum_{t=h_{2}}^{\infty} \delta^{t-h}\right) a_{*}^{h_{2}, \delta} .
$$

We assume that the sequence $a_{1}, a_{2}, \ldots$ is bounded by $M \geq 1$. Since $a$ is an accumulation point, there are $h_{1}<h_{2}$ such that

$$
\sum_{t=h_{1}}^{h_{2}-1} \delta^{t-h_{1}}>1-\frac{\gamma}{2 M} \text { and }\left|a_{*}^{h, \delta}-a\right|<\frac{\gamma}{2}, \text { for } h=h_{1}, h_{2} .
$$

We consider the greatest $h, h_{1} \leq h \leq h_{2}$ such that $a_{h} \geq a-\gamma$. There exists such $h$ because if all $h$ between $h_{1}$ and $h_{2}$ satisfy $a_{h}<a-\gamma$, then

$$
a_{*}^{h_{1}}<\left(1-\frac{\gamma}{2 M}\right)(a-\gamma)+\frac{\gamma}{2 M} M<a-\frac{\gamma}{2},
$$


which contradicts the choice of $h_{1}$.

As for $a_{*}^{h}$, (5.4) applied to $h=h_{1}$ and to $h_{2}$ implies that

$$
\begin{aligned}
a_{*}^{h, \delta} & =(1-\delta) \sum_{t=h}^{h_{2}-1} \delta^{t-h} a_{t}+(1-\delta)\left(1-\sum_{t=h_{2}}^{\infty} \delta^{t-h}\right) a_{*}^{h_{2}, \delta} \\
& \leq(1-\delta) a_{h}+(1-\delta) \sum_{t=h+1}^{h_{2}-1} \delta^{t-h}(a-\gamma)+(1-\delta)\left(1-\sum_{t=h_{2}}^{\infty} \delta^{t-h}\right)(a+\gamma / 2)
\end{aligned}
$$

When $\delta$ is large enough, $(1-\delta) a_{h}<\gamma / 2$. Thus, $a_{*}^{h, \delta} \leq \gamma / 2+\delta(a+\gamma / 2) \leq a+\gamma$, as desired.

Proof of Theorem 5.6. We assume without loss of generality that $v_{1}(N)=1$. We prove that (ii) implies (ii). We assume that for any $\varepsilon>0$ the $\varepsilon$-stable core of $(V, \delta)$ is not empty for $\delta$ sufficiently large. Fix $\varepsilon>0$ and assume that $1-\varepsilon<\delta$.

Let $m$ be the one guaranteed by Lemma 5.5 and $\varepsilon$. Suppose that the discount factor $\delta$ is large enough so the total payoff of any coalition during $m$ periods could not exceed $\varepsilon$.

Let $x_{1}, x_{2}, \ldots$ be in the $\varepsilon$-stable core of $(V, \delta)$ and let $x_{*}$ be an accumulation point of the sequence $x_{*}^{h}=x_{*}^{h}(\delta)=(1-\delta) \sum_{t=h}^{\infty} \delta^{t-h} x_{t}, h=1,2 \ldots$ (it exists because, by assumption, the sequence of allocations is bounded).

By assumption, $x_{1}(N)=x_{2}(N)=\ldots$ We will show that $x_{*}$ is in the $5 \varepsilon$-core of $u_{x_{*}}$. If not, then there is a coalition $T$ such that $x_{*}(T)<u_{x_{*}}(T)-5 \varepsilon$. In particular, $x_{*}(T)$ is not a fixed point of $f_{T}$. Since for every time $h, x_{*}^{h}$ is an average of $x_{h}, x_{h+1}, \ldots$, we have that $x(N):=x_{*}^{h}(N)=V\left(N, x_{t}\right)(N)=v_{1}(N)$ (the last equality is by assumption) for every period $t$, implying that $x_{*}$ is an allocation of $v_{1}$.

Since the set of fixed points of $f_{T}$ is closed, and $x_{*}(T)$ is not a fixed point of $f_{T}$, one can find $\beta>0$ such that

$$
x_{*}^{h}(T)>x_{*}(T)-\beta \text { implies } f_{T}\left(x_{*}^{h}(T)\right) \geq f_{T}\left(x_{*}(T)\right) .
$$

The reason is that when $x_{*}^{h}(T)$ is not a fixed point of $f_{T}$, there is an open interval around $x_{*}^{h}(T)$, whose points have all the same range as $x_{*}^{h}(T)$. That is, $f_{T}$ is fixed around $x_{*}^{h}(T)$. Thus, there is $\beta>0$ such that $x_{*}^{h}(T)>x_{*}(T)-\beta$ implies $f_{T}\left(x_{*}^{h}(T)\right)=$ $f_{T}\left(x_{*}(T)\right)$. In case $x_{*}^{h}(T)>x_{*}(T)$, then by monotonicity $f_{T}\left(x_{*}^{h}(T)\right) \geq f_{T}\left(x_{*}(T)\right)$ and therefore, (5.5).

Applying Lemma 5.7 to the sequence $x_{1}(T), x_{2}(T), \ldots$ and the accumulation point $x_{*}(T)$, we obtain that for $\gamma=\min (\beta, \varepsilon)$, there is a time $h$ such that

$$
x_{h}(T)>x_{*}(T)-\gamma \text { and } x_{*}^{h}(T)<x_{*}(T)+\gamma
$$

In words, the instantaneous payoffs of coalition $T$ at time $h$ is greater than $x_{*}(T)-\gamma$, while the present value of coalition T's payoff at time $h+1$ is less than $x_{*}(T)+\gamma$. 
We now describe a deviation of coalition $T$. At time $h+1$ coalition $T$ deviates and plays the game $V\left(T, x_{h}(T)\right)$. From time $h+2$ on, any allocation of the stage-game is fine. After $m$ periods the worth of the coalition $T$ in the stage-game, is by Lemma 5.5, close to $u_{x_{h}}(T)$ up to $\varepsilon$. Since the first $m$ periods after $h$ contribute at most $\varepsilon$ to the entire present value of coalition $T$, the payoff (discounted to time $h+1$ ) for $T$ due to the deviation is at least $u_{x_{h}}(T)-2 \varepsilon$.

By (5.6), $x_{h}(T)>x_{*}(T)-\gamma \geq x_{*}(T)-\beta$. Because of (5.5), $u_{x_{h}}(T) \geq u_{x_{*}}(T)$. Thus, due to the deviation, the payoff of coalition $T$ is at least $u_{x_{*}}(T)-2 \varepsilon$. However, the sequence $x_{1}, x_{2}, \ldots$ is in the $\varepsilon$-stable core of $(V, \delta)$, and therefore, by deviating coalition $T$ cannot get more than $\varepsilon$ beyond the originally planned payoff $\left(x_{*}^{h+1}(T)\right)$. Thus,

$$
u_{x_{*}}(T)-2 \varepsilon \leq x_{*}^{h+1}(T)+\varepsilon .
$$

Again from (5.6) we have $x_{*}^{h}(T)<x_{*}(T)+\gamma \leq x_{*}(T)+\varepsilon$. Thus,

$$
x_{*}^{h+1}(T) \leq x_{*}^{h}(T)+\varepsilon \leq x_{*}(T)+2 \varepsilon .
$$

The first inequality of (5.8) is due to the fact that $1-\delta<\varepsilon$ (as assumed at the beginning of the proof) and $x_{h}(T) \leq v_{1}(N)=1$. Hence, by (5.7) and (5.8), $u_{x_{*}}(T)-2 \varepsilon \leq x_{*}^{h+1}(T)+\varepsilon \leq x_{*}(T)+3 \varepsilon$. It implies that $u_{x_{*}}(T)-5 \varepsilon \leq x_{*}(T)$. This is a contradiction, and therefore $x_{*}$ is the $5 \varepsilon$-core of $u_{x_{*}}$.

The proof that (iii) implies (ii) is relatively easy and is therefore omitted.

Remark 5.8. Assertion (iii) of Theorem 5.6 states that for every $\varepsilon>0$ there exists an allocation $x$ of $v_{1}$ such that the $\varepsilon$-core of $u_{x}$ is not empty. Due to lack of continuity it is impossible to conclude that there exists an allocation $x$ of $v_{1}$ such that the core of $u_{x}$ is not empty. This is so because when a sequence of allocations of $v_{1}$, say $\left(x_{k}\right)_{k}$ (each in the $\varepsilon$-core of the respective $u_{x_{k}}$ ), is converging to $x$, there is no guarantee that $u_{x_{k}}$ converges to $u_{x}$.

\section{The credible core}

The third type of core we are about to define is close in spirit to subgame perfect equilibrium in the theory of non-cooperative games. A dissatisfied coalition may deviate at any time in which future allocations guarantee less than it can do alone. Hence, stability conditions must be preserved not only at the beginning of the game, but throughout the entire game. But when creating its own game, a coalition, say $S$ may face a threat from one of its sub-coalitions, say $T$. The game established by $T$ may depend on the entire history of allocations, starting from the grand coalition allocation at the beginning of game, continuing with $S$-allocation and ending with allocations of its own.

The game may start with the grand coalition, run this way for a while and only then coalition $S_{1}$ may decide to deviate, run for a while and then coalition $S_{2}$ may 
deviate etc. Thus, histories now consist of $x_{1}^{S_{1}}, x_{2}^{S_{2}}, \ldots, x_{t}^{S_{t}}$, where $S_{1}=N$ and the sequence of $S_{\ell}$ is decreasing (w.r.t. inclusion). A history $x_{1}^{S_{1}}, x_{2}^{S_{2}}, \ldots, x_{t}^{S_{t}}$ is feasible if at any time $t, x_{t}^{S_{t}}$ is an allocation of the stage game $V^{S_{t}}\left(x_{t-1}^{S_{t-1}}\right)$.

A central planner has an allocation policy, denoted $\sigma$, that associates with any time and any possible instantaneous game an allocation. In other words, the central planner has a full contingency plan as to how the available cake should be split at any point in time. Formally, the allocation policy $\sigma$ is such that $\sigma\left(x_{t}^{S_{t}}\right)$ is an allocation of the game $V\left(S_{t+1} ; x_{t}^{S_{t}}\right)$.

After any history of allocations, $x_{1}^{S_{1}}, x_{2}^{S_{2}}, \ldots, x_{t}^{S_{t}}$, an allocation policy $\sigma$ determines uniquely a continuation stream of allocations: $x_{t+1}^{S_{t}}=\sigma\left(x_{t}^{S_{t}}\right), x_{t+2}^{S_{t}}=\sigma\left(x_{t+1}^{S_{t}}\right)$, etc. We denote this continuation by $C_{\sigma}\left(x_{1}^{S_{1}}, x_{2}^{S_{2}}, \ldots, x_{t}^{S_{t}}\right)$.

Definition 6.1 (Credible core). An allocation policy $\sigma$ is in the credible core with discount factor $\delta$ of $(V, \delta)$ if for every history $x_{1}^{S_{1}}, x_{2}^{S_{2}}, \ldots, x_{t}^{S_{t}}$ the sequence $C_{\sigma}\left(x_{1}^{S_{1}}, x_{2}^{S_{2}}, \ldots, x_{t}^{S_{t}}\right)$ of allocations is in the stable core of $V\left(S_{t+1} ; x_{t}^{S_{t}}\right)$.

A similar idea has been used in a non-cooperative context by Bernheim et al. (1987); Bernheim and Whinston (1987). These authors consider Nash equilibria that are immune from deviations not just of single players but also of coalitions. Not every deviation is acceptable, though: a deviation of some coalition has to be in turn immune from deviations of sub-coalitions.

The main result of this section shows that a form of the one-deviation principle holds for the credible core. This principle goes back to Blackwell (1965) and has been widely used in extensive form noncooperative games. Recently Vartiainen (2008) applied it in the study of coalition formation in a cooperative context.

Definition 6.2. A coalition $S \varsubsetneqq S_{t}$ has a profitable one-deviation after the history $x_{1}^{S_{1}}, x_{2}^{S_{2}}, \ldots, x_{t}^{S_{t}}$ from $\sigma$, if there is an allocation $x_{t+1}^{S}$ of $V\left(S ; x_{t}^{S_{t}}\right)$ such that the present value for coalition $S$ of the sequence that starts at $x_{t+1}^{S}$ and continues with $C_{\sigma}\left(x_{1}^{S_{1}}, x_{2}^{S_{2}}, \ldots, x_{t}^{S_{t}}, x_{t+1}^{S}\right)$ is greater than the present value for $S$ of the planned sequence $C_{\sigma}\left(x_{1}^{S_{1}}, x_{2}^{S_{2}}, \ldots, x_{t}^{S_{t}}\right)$.

Theorem 6.3 (The one-deviation principle). An allocation policy $\sigma$ is in the credible core if and only if after every history $x_{1}^{S_{1}}, x_{2}^{S_{2}}, \ldots, x_{t}^{S_{t}}$ and for every $S \subsetneq S_{t}$ there is no profitable one-deviation.

Proof. The 'only if' direction is trivial. For the 'if' part, assume that after every history $x_{1}^{S_{1}}, x_{2}^{S_{2}}, \ldots, x_{t}^{S_{t}}$ and for every $S \subsetneq S_{t}$ there is no profitable one-deviation. Suppose that there is a coalition $S \subsetneq S_{t}$ that has a profitable deviation after the sequence $x_{1}^{S_{1}}, x_{2}^{S_{2}}, \ldots, x_{t}^{S_{t}}$. Denote the gain by $a>0$. By continuity we may assume that this deviation consists of finite number stages. Consider the shortest deviation of $S$ after $x_{1}^{S_{1}}, x_{2}^{S_{2}}, \ldots, x_{t}^{S_{t}}$ that guarantees a gain of at least $a$. This deviation is $x_{t+1}^{S}, \ldots, x_{t+\ell}^{S}$. It implies that the deviation $x_{t+1}^{S}, \ldots, x_{t+\ell-1}^{S}$ guarantees $S$ less than $a$, meaning that the single deviation of $S$ to $x_{t+\ell}^{S}$ after the entire history 
$x_{1}^{S_{1}}, x_{2}^{S_{2}}, \ldots, x_{t}^{S_{t}}, x_{t+1}^{S}, \ldots, x_{t+\ell-1}^{S}$ makes a positive gain for $S$. This shows that there is no profitable one-deviation, as this direction of the theorem claims.

Note that the theorem does not say that when the allocation policy $\sigma$ is in the credible core, the instantaneous allocations are in the respective stage game.

Remark 6.4. Theorem 6.3 holds also under a richer non-Markovian dynamic, where $V$ depends on the entire history of allocations and not only on the last allocation.

\section{$7 \quad$ Final remarks}

We close the paper with some additional comments.

\section{More on the non-emptyness of the core}

Consider a game where $V(N ; \cdot)(N)$ is constant. In addition assume that for every coalition $S, V(N ; \cdot)(S)$ is bounded. The $\varepsilon$-stable core with discount factor $\delta$ is not empty if and only if the core of $v_{*}(\cdot, \delta)$ is non-empty.

\section{No-short assumption}

Throughout the paper we assumed that the stage allocation, $x_{t}^{S}$, satisfies two assumptions. First, the allocation of player $i$ is at least her $v(i)$, that is, $x_{t}^{S}(i) \geq B$, and second, $x_{t}^{S}$ is locally efficient, that is, $x_{t}^{S}(S)=v_{t}^{S}(S)$. This assumption assumes that the inter-temporal transfers are limited. That is $B$ might be well below $v_{t}(i)$ at a certain moment, but since the overall payoff in the entire dynamic game needs to be individually rational, the instantaneous payoffs need to be sometimes higher than the stage IR level. The technical advantage of these assumptions is that the set of possible allocations at any stage is compact.

\section{Random games}

We analyze games where the instantaneous game depends deterministically on the history. The issue of stochastic dynamic game where the stage games are endogenously determined remains open for further studies.

\section{Acknowledgements}

The authors thank Sandro Brusco for helpful comments and relevant references. 


\section{References}

Becker, R. A. and Chakrabarti, S. K. (1995) The recursive core. Econometrica 63, 401-423.

Bernheim, B. D., Peleg, B., and Whinston, M. D. (1987) Coalitionproof Nash equilibria. I. Concepts. J. Econom. Theory 42, 1-12. URL http://dx.doi.org/10.1016/0022-0531(87)90099-8.

Bernheim, B. D. and Whinston, M. D. (1987) Coalition-proof Nash equilibria. II. Applications. J. Econom. Theory 42, 13-29. URL http://dx.doi.org/10.1016/0022-0531(87)90100-1.

Blackwell, D. (1965) Discounted dynamic programming. Ann. Math. Statist. 36, $226-235$.

Chwe, M. S.-Y. (1994) Farsighted coalitional stability. J. Econom. Theory 63, 299-325. URL http://dx.doi.org/10.1006/jeth.1994.1044.

Diamantoudi, E. and Xue, L. (2007) Coalitions, agreements and efficiency. $J$. Econom. Theory 136, 105-125.

FudenberG, D. and Maskin, E. (1986) The folk theorem in repeated games with discounting or with incomplete information. Econometrica 54, 533-554.

Gale, D. (1978) The core of a monetary economy without trust. J. Econom. Theory 19, 456-491.

Habis, H. and Herings, P. J.-J. (2010) A Note on The Weak Sequential Core of Dynamic TU Games. Research Memoranda 022, Maastricht : METEOR, Maastricht Research School of Economics of Technology and Organization. URL http://econpapers.repec .org/RePEc: dgr : umamet :2010022.

Hellman, Z. (2008) Bargaining set solution concepts in dynamic cooperative games. Technical report, Munich Personal RePEc Archive. URL http://mpra.ub.uni-muenchen.de/8798/.

Konishi, H. and RAY, D. (2003) Coalition formation as a dynamic process. $J$. Econom. Theory 110, 1-41.

Koutsougeras, L. C. (1998) A two-stage core with applications to asset market and differential information economies. Econom. Theory 11, 563-584.

Kovalenkov, A. and Wooders, M. (2003) Approximate cores of games and economies with clubs. J. Econom. Theory 110, 87-120. 
Kovalenkov, A. and Wooders, M. (2005) Laws of scarcity for a finite game exact bounds on estimations. Econom. Theory 26, 383-396. URL http://dx.doi.org/10.1007/s00199-003-0443-7.

Kovalenkov, A. and Wooders, M. H. (2001a) Epsilon cores of games with limited side payments: nonemptiness and equal treatment. Games Econom. Behav. 36, 193-218. URL http://dx.doi.org/10.1006/game.2000.0815.

Kovalenkov, A. and Wooders, M. H. (2001b) An exact bound on epsilon for nonemptiness of epsilon cores of games. Math. Oper. Res. 26, 654-678. URL http://dx.doi.org/10.1287/moor.26.4.654.10001.

Kranich, L., Perea, A., and Peters, H. (2005) Core concepts for dynamic TU games. Int. Game Theory Rev. 7, 43-61.

Oviedo, J. (2000) The core of a repeated $n$-person cooperative game. European J. Oper. Res. 127, 519-524.

Petrosjan, L. and Zaccour, G. (2003) Time-consistent Shapley value allocation of pollution cost reduction. J. Econom. Dynam. Control 27, 381-398.

Petrosjan, L. A. (1977) Stability of the solutions in differential games with several players. Vestnik Leningrad. Univ. 46-52, 147.

Petrosjan, L. A. (1993) Differential Games of Pursuit. World Scientific Publishing Co. Inc., River Edge, NJ.

Predtetchinski, A. (2007) The strong sequential core for stationary cooperative games. Games Econom. Behav. 61, 50-66.

Predtetchinski, A., Herings, P. J. J., and Perea, A. (2006) The weak sequential core for two-period economies. Internat. J. Game Theory 34, 55-65.

Predtetchinski, A., Herings, P. J.-J., and Peters, H. (2002) The strong sequential core for two-period economies. J. Math. Econom. 38, 465-482.

Predtetchinski, A., Herings, P. J.-J., and Peters, H. (2004) The strong sequential core in a dynamic exchange economy. Econom. Theory 24, 147-162.

RAY, D. (1989) Credible coalitions and the core. Internat. J. Game Theory 18, 185-187. URL http://dx.doi.org/10.1007/BF01268157.

Ray, D. (2007) A Game-Theoretic Perspective on Coalition Formation. Lipsey Lectures. Oxford University Press, Oxford.

RAY, D. and VoHra, R. (1999) A theory of endogenous coalition structures. Games Econom. Behav. 26, 286-336. URL http://dx.doi.org/10.1006/game.1998.0648. 
Shapley, L. S. and ShubiK, M. (1966) Quasi-Cores in a Monetary Economy with Nonconvex Preferences. Econometrica 34, pp. 805-827. URL http://www.jstor.org/stable/1910101.

Shubik, M. and Wooders, M. H. (1983a) Approximate cores of replica games and economies. I. Replica games, externalities, and approximate cores. Math. Social Sci. 6, 27-48. URL http://dx.doi.org/10.1016/0165-4896(83)90044-6.

ShubIK, M. and Wooders, M. H. (1983b) Approximate cores of replica games and economies. II. Set-up costs and firm formation in coalition production economies. Math. Social Sci. 6, 285-306. URL http://dx.doi.org/10.1016/0165-4896(83)90063-X.

VARtiainen, H. (2008) One-deviation principle in coalition formation. Discussion Papers 35, Aboa Centre for Economics. URL http://ideas.repec.org/p/tkk/dpaper/dp35.html.

Wooders, M. H. (1983) The epsilon core of a large replica game. J. Math. Econom. 11, 277-300. URL http://dx.doi.org/10.1016/0304-4068(83)90005-8.

Wooders, M. H. and Zame, W. R. (1984) Approximate cores of large games. Econometrica 52, 1327-1350. URL http://dx.doi.org/10.2307/1913508.

Xue, L. (1998) Coalitional stability under perfect foresight. Econom. Theory 11, 603-627. URL http://dx.doi.org/10.1007/s001990050204. 